\title{
RHIC AND THE SEARCH FOR HIGH ENERGY DENSITY MATTER
}

\author{
LARRY MCLERRAN \\ Physics Department \\ PO Box 5000 Brookhaven National Laboratory \\ Upton, NY 11973 USA
}

\begin{abstract}
The major goal of the RHIC experimental program at Brookhaven National Laboratory is to make and study the Quark Gluon Plasma. Another new form of matter, the Color Glass Condensate may be formed in these collisions The recent results from RHIC are reviewed in this context.
\end{abstract}

\section{High Density Matter: The Frontier of High Energy Nuclear Physics}

\subsection{The Goals of RHIC}

The goal of nuclear physics has traditionally been to study matter at the highest possible densities. This has been done in the past by studying the atomic nucleus, where energy densities become of the order of

$$
\epsilon \sim .15 \mathrm{GeV} / \mathrm{Fm}^{3}
$$

High energy nuclear physics has extended this study to energy densities several orders of magnitude higher. This extension includes the study of matter inside ordinary strongly interacting particles, such as the proton and the neutron, and producing new forms of matter at much higher energy densities in high energy collisions of nuclei with nuclei, and various other probes.

RHIC is a multi-purpose machine which can address at least three central issues of high energy nuclear physics. These are:

- The production of matter at energy densities one to two orders of magnitude higher than that of nuclear matter and the study of its properties.

This matter is at such high densities that it is only simply described in terms of quarks and gluons and is generically referred to as the 
Quark Gluon Plasma. The study of this matter may allow us to better understand the origin of the masses of ordinary particles such as nucleons, and of the confinement of quarks and gluons into hadrons. The Quark Gluon Plasma will be described below.

- The study of the matter which controls high energy strong interactions.

This matter is believed to be universal (independent of the hadron), and exists over sizes enormous compared to the typical microphysics size scales important for high energy strong interactions. (The microphysics time scale here is roughly the size scale here is about $1 \mathrm{Fm}$ and the microphysics time scale is the time it takes light to fly $1 F m, t \sim 10^{-23} \mathrm{sec}$.) It is called a Color Glass Condensate because it is composed of colored particles, gluons, evolves on time scales long compared to microphysics time scales and therefore has properties similar to glasses, and a condensate since the phase space density of gluons is very high. The study of this matter may allow us to better understand the typical features of strong interactions when they are truly strong, a problem which has eluded a basic understanding since strong interactions were first discovered. The Color Glass Condensate will be described below.

- The study of the structure of the proton, most notably spin.

Because of the subject of this meeting, I shall discuss only the first two issues.

\subsection{The Quark Gluon Plasma}

This section describes what is the Quark Gluon Plasma, why it is important for astrophysics and cosmology, and why it provides a laboratory in which one can study the origin of mass and of confinement.?

\subsubsection{What is the Quark Gluon Plasma?}

Matter at low energy densities is composed of electrons, protons and neutrons. If we heat the system, we might produce thermal excitations which include light mass strongly interacting particles such as the pion. Inside the protons, neutrons and other strongly interacting particles are quarks and gluons. If we make the matter have high enough energy density, the 
protons, nucleons and other particles overlap and get squeezed so tightly that their constituents are free to roam the system without being confined inside hadrons. At this density, there is deconfinement and the system is called a Quark Gluon Plasma. This is shown in Fig. 1

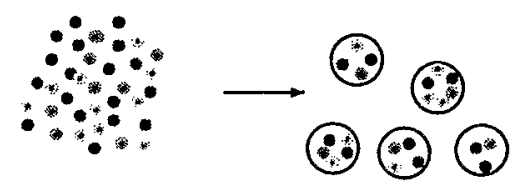

\section{Quark-Gluon Plasma $\longrightarrow$ Hadron Gas}

Figure 1. As the energy density is decreased, the Quark Gluon Plasma condenses into a low density gas of hadrons. Quarks are red, green or blue and gluons are yellow.

As the energy density gets to be very large, the interactions between the quarks and gluons become weak. This is a consequence of the asymptotic freedom of strong interactions: At short distances the strong interactions become weak.

\subsubsection{The Quark Gluon Plasma and Fundamental Physics Issues}

The nature of matter at high densities is an issue of fundamental interest. Particle physics defines a problem of fundamental interest as exclusively the determination of the properties of the first principles Hamiltonian which describes all of particle interactions. For nuclear physics, this issue is different since this field is interested in the properties of matter as it might appear in nature. Fundamental here means that one understands the basic behaviour of this matter and the diversity of forms in which it can appear For example: the structure of a proton or a nucleus. In the case of the quark gluon plasma: the properties of matter in bulk. Of course matter in bulk can have a much richer structure than is easily guessed from the fundamental Hamiltonian. Maxwell's equations together with the Schrodinger equation are indeed very simple but in fact predict the wide and complex structure of all forms of ordinary matter.

A hypothetical phase diagram for QCD is shown in Fig. 2. The vertical axis is temperature, and the horizontal is a measure of the matter or baryon number density, the baryon number chemical potential. The solid lines indicate a first order phase transition, and the dashed line a rapid cross over. It is not known whether or not the region marked cross over is or is 
not a true first order phase transition. There are analytic arguments for the properties of matter at high density, but numerical computation are of insufficient resolution. At high temperature and fixed baryon number density, there are both analytic arguments and numerical computations of good quality. At high density and fixed temperature, one goes into a superconducting phase, perhaps multiple phases of superconducting quark matter. At high temperature and fixed baryon number density, the degrees of freedom are those of a Quark Gluon Plasma.

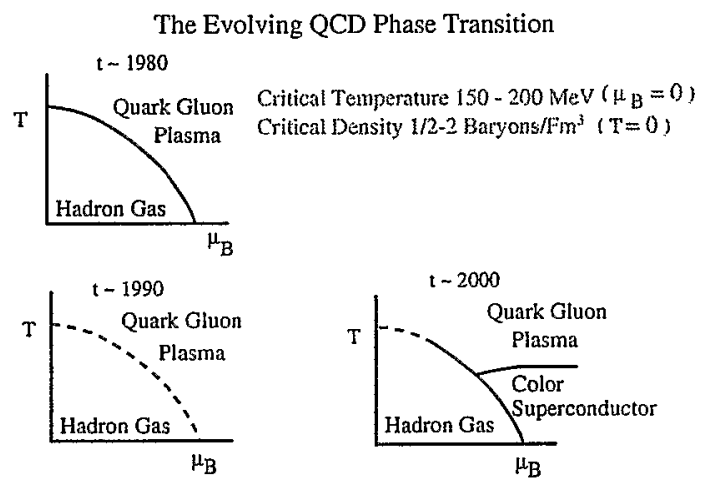

Figure 2. A phase diagram for QCD collisions.

Lattice gauge theory numerical studies, and analytic studies have taught us us much about the properties of these various phases of matter.? At high energy densities, the linear confining force disappears, and chiral symmetry is restored, which means the masses for quarks which are generated at low energy density become small. The study of these various phases is the subject of much of this meeting.

\subsection{The Color Glass Condensate}

This section describes what is the Color Glass Condensate, and why it is important for our understanding of basic properties of strong interactions.? I argue that the Color Glass Condensate is a universal form of matter which controls the high energy limit of all strong interaction processes and is the part of the hadron wavefunction important at such energies. Since the Color Glass Condensate is universal and controls the high energy limit of all strong interactions, it is of fundamental importance. 


\subsubsection{What is the Color Glass Condensate?}

A very high energy hadron has contributions to its wavefunction from gluons, quarks and anti-quarks with energies up to that of the hadron and all the way down to energies of the order of the scale of light mass hadron masses, $E \sim 200 \mathrm{MeV}$. A convenient variable in which to think about these quark degrees of freedom is the typical energy of a constituent scaled by that of the hadron,

$$
x=E_{\text {constituent }} / E_{\text {hadron }}
$$

Clearly the higher the energy of the hadron we consider, the lower is the minimum $x$ of a constituent. Sometimes it is also useful to consider the rapidity of a constituent which is $y \sim \ln (1 / x)$

The density of small $x$ partons is

$$
\frac{d N}{d y}=x G\left(x, Q^{2}\right)
$$

The scale $Q^{2}$ appears because the number of constituents one measures depends (weakly) upon the resolution scale of the probe with which one measures. (Resolution scales are measured in units of the inverse momentum of the probe, which is usually taken to be a virtual photon.) A plot of $x G\left(x, Q^{2}\right)$ for gluons at various $x$ and $Q^{2}$ measured at the HERA accelerator in protons is shown in Fig. 3.

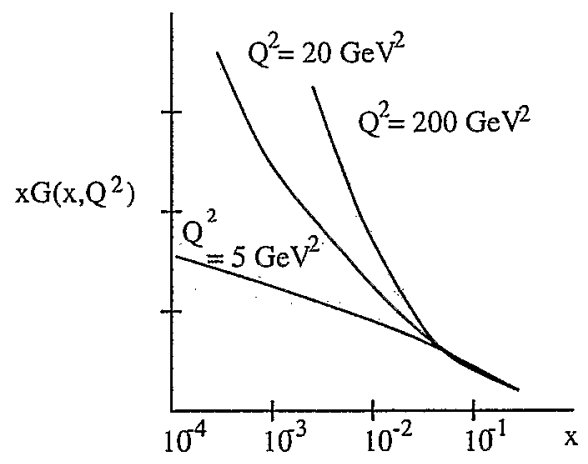

Figure 3. The number of gluons in a proton per unit rapidity at various rapidities and $Q^{2}$ resolutions.

Note that the gluon density rises rapidly at small $\mathrm{x}$ in Fig. 3. This is the so called small $x$ problem. It means that if we view the proton head 
on at increasing energies, the low momentum gluon density grows. This is shown in Fig. 4.

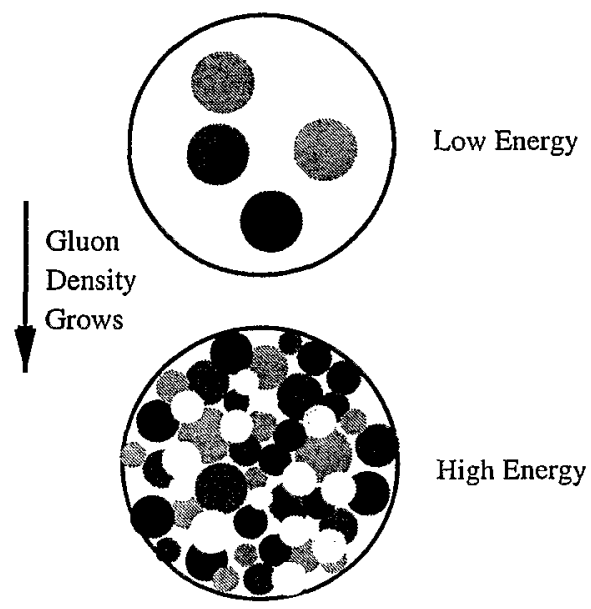

Figure 4. The increasing density of wee partons as the energy increases.

As the density of gluons per unit area, per unit rapidity increases, the typical transverse separation of the gluons decreases. This means that the matter which controls high energy strong interactions is very dense, and it means that the QCD interaction strength, which is usually parameterized by the dimensionless scale $\alpha_{S}$ becomes small. The phase space density of these gluons, $\rho \sim 1 / \pi R^{2} d N / d^{2} p_{T}$ can become at most $1 / \alpha_{s}$ since once this density is reached gluon interactions are important. This is characteristic of Bose condensation phenomena which are generated by an instability proportional to the density $\rho$ and is compensated by interactions proportional to $\alpha_{S} \rho^{2}$, which become of the same order of magnitude when $\rho \sim 1 / \alpha_{s}$ Thus the matter is a Color Condensate.

The glassy nature of the condensate arise because the fields associated with the condensate are generated by constituents of the proton at higher momentum. These higher momentum constituents have their times scales Lorentz time dilated relative to those which would be measured in their rest frame. Therefore the fields associated with the low momentum constituents also evolve on this long time scale. The low momentum constituents are therefore glassy: their time evolution scale is unnaturally long compared to their natural time scale. Hence the name Color Glass Condensate. 
There is also a typical scale associated with the Color Glass Condensate: the saturation momentum. This is the typical momentum scale where the phase space density of gluons becomes $\rho<<1 / \alpha_{S}$.

At very high momentum, the fields associated with the Color Glass Condensate can be treated as classical fields, like the fields of electricity and magnetism. Since they arise from fast moving partons, they are plane polarized, with mutually orthogonal color electric and magnetic fields perpendicular to the direction of motion of the hadron. They are also random in two dimensions. This is shown in Fig. 5.

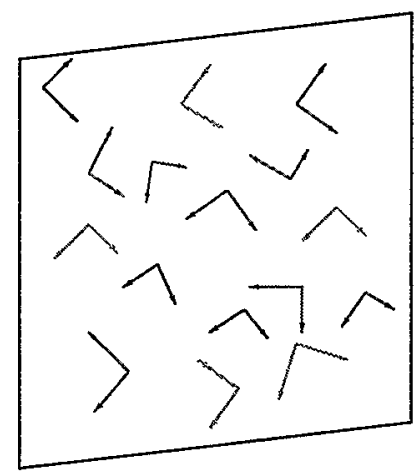

Figure 5. The Color Glass Condensate as a high density of random gluon fields on a two dimensional sheet traveling near the speed of light.

\subsubsection{Why is the Color Glass Condensate Important?}

The Color Glass Condensate is the universal form of matter from which all high energy hadrons are made. Like nuclei and electrons compose atoms, and nucleons and protons compose nuclear matter, the Color Glass Condensate is the fundamental matter of which high energy hadrons are composed.

The Color Glass Condensate has the potential to allow for a first principles description of the gross or typical properties of matter at high energies. For example, the total cross section at high energies for proton-proton scattering, as shown in Fig. 6 has a simple form but for over 40 years has resisted simple explanation. (It has perhaps been recently understood in terms of the Color Glass Condensate or Saturation ideas.)

The Color Glass Condensate form the matter in the quantum mechanical state which describes a nucleus. In the earliest stages of a nucleus- 
The total hadronic cross section:

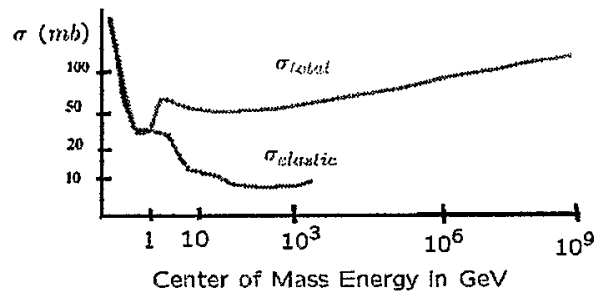

Figure 6. The total cross section for high energy proton-proton interactions.

nucleus collisions, the matter must not be changed much from these quantum mechanical states. The Color Glass Condensate therefore provides the initial conditions for the Quark Gluon plasma to form in these collisions. A space-time picture of nucleus nucleus collisions is shown in Fig. 7. At very early times, the Color Glass Condensate evolves into a distribution of gluons. Later these gluons thermalize and may eventually form a Quark Gluon Plasma. At even later times, a mixed phase of plasma and hadronic gas may form.

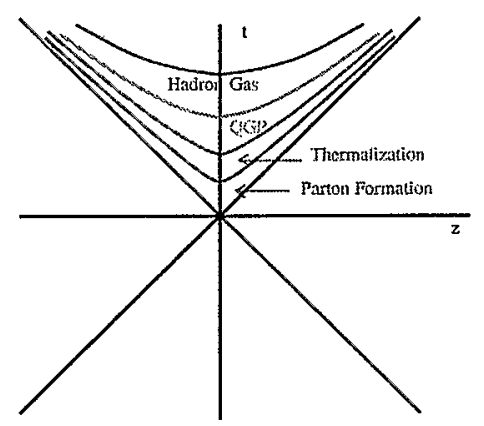

Figure 7. A space-time diagram for the evolution of matter produced in heavy ion collisions.

\section{How Do Heavy Ion Collisions at RHIC Address the Scientific Issues?}

Heavy ion collisions at ultrarelativistic energies are visualized in Fig. 8 as the collision of two sheets of colored glass. 
At ultrarelativistic energies, these sheets pass through one another. In their wake is left melting colored glass, which eventually materializes as quarks and gluons. These quarks and gluons would naturally form in their restframe on some natural microphysics time scale. For the saturated color glass, this time scale is of order the inverse saturation momentum (again, we convert momentum into time by appropriate uses of Planck's constant and the speed of light), in the rest frame of the produced particle. When a particle has a large momentum along the beam axis, this time scale is Lorentz dilated. This means that the slow particles are produced first towards the center of the collision regions and the fast particles are produced later further away from the collision region. This correlation between space
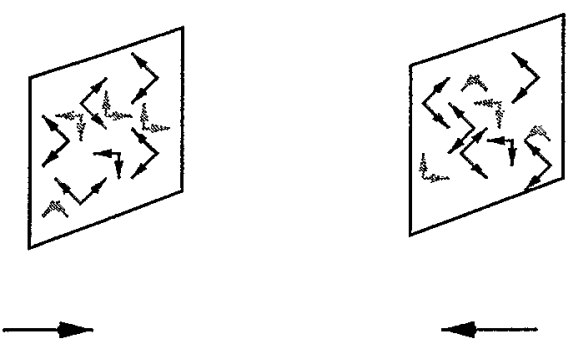

Figure 8. The collision of two sheets of colored glass.

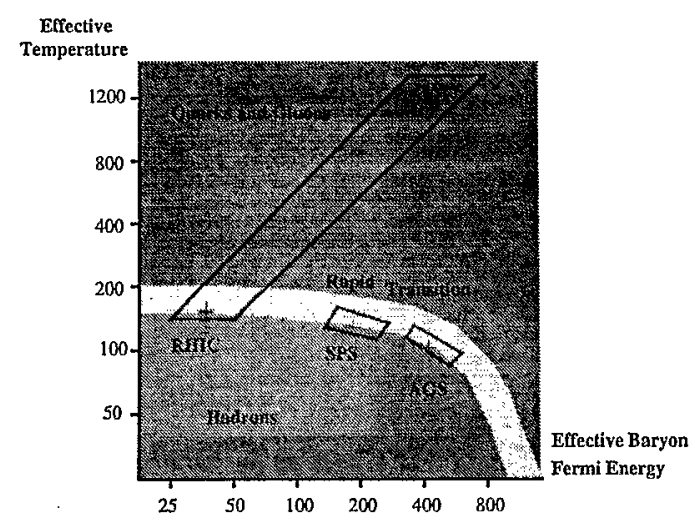

Figure 9. The reach of RHIC.

and momentum is similar to what happens to matter in Hubble expansion 
in cosmology. The stars which are further away have larger outward velocities. This means that this system, like the universe in cosmology is born expanding.

As this system expands, it cools. Presumably at some time the produced quarks and gluons thermalize, They then expand as a quark gluon plasma and eventually as some mixture of hadrons and quarks and gluons. Eventually, they may become a gas of only hadrons before they stop interacting and fly off to detectors.

One can estimate the reach of RHIC compared to previous machines by the Fig. 9. On this Figure, we attempt to estimate the typical energy densities and baryon number densities by an effective temperature and effective baryon number chemical potential (a measure of the baryon number density). (In the next section, we discuss how the temperature and baryon chemical potential are determined by the RHIC experiments.) For nonthermal systems, the effective temperature would be proportional to the typical energy per particle in the rest frame of the moving system, and is defined so that it reduces to the temperature for a thermal system. The baryon chemical potential is defined so that the ratio of effective baryon chemical potential to effective temperature determines the baryon number to entropy ratio in the same way it would for thermal systems.

As can be seen from the figure, the effective temperature scales one can achieve at RHIC are enormous compared to those appropriate for the transition from hadronic degrees of freedom to those of quarks and gluons.

We make this case clear in the next section: RHIC makes QuarkGluon Matter. About that there is now little doubt. The questions which must be answered are about the nature of this matter. What are its properties? Is it thermalized? Does it remember its origin as a Color Glass Condensate? Can one use these collisions as a laboratory to study the generations of mass or the confinement of quarks and gluons?

\section{What We Have Learned from RHIC}

In this section, we discuss the results from RHIC and what this implies about the search for new forms of matter. We will concentrate on issues related to the Quark Gluon Plasma and to the Color Glass Condensate.

We shall also describe in what directions the recent results from RHIC are driving future directions. It may seem a little confusing the different directions which experimentalists are pursuing. It must be understood that science after all progresses not as a linear process or entirely logical process. 
There is an old joke which illustrates this and which has it origin in an old story by the famous Russian theorist Gribov. A man is lost in the forest and his home is some distance away. This is shown in Fig. 10. Being

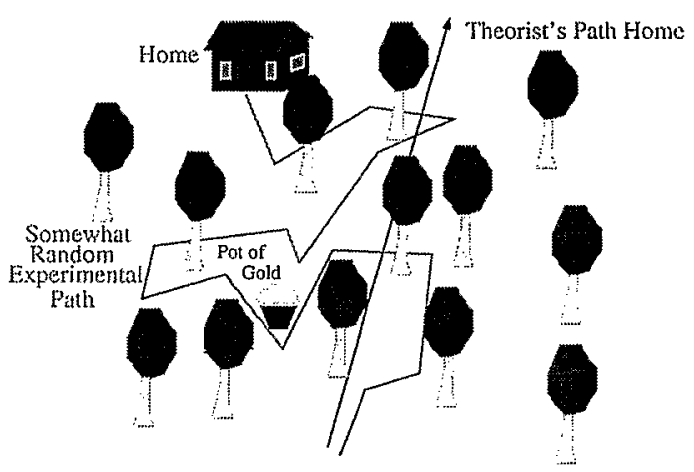

Figure 10. Gribov's walk home.

lost in the forest is analogous to making a random walk in two dimensions: You go a little ways, change directions go further, change directions again, etc. etc. Now in two space dimensions there is a theorem that a random walk guarantees that the person will always eventually find his way home, but it may take a long time. An experimentalist on the other hand knows roughly where his home is located, and makes random walks in the general direction of home, and will arrive there in a much quicker time. How can one fail to get home? If the person is a theorist, they might think they know exactly the direction to home! They begin walking in that direction, miss the house but continue on because they are certain of their direction. The experimentalist has the added advantage relative to the theorist that in his directed random walk, he may discover something unexpected in his excursion from the shortest path.

The situation at RHIC is somewhat like this. We are exploring new territory, and hopefully in addition to understanding properties of the Quark Gluon Plasma. We know roughly how to proceed, but since this is a new frontier, there are no road maps. It already seems we have stumbled on to something unexpected in the RHIC experiments. There is increasing evidence that one is finding effects associated with parton saturation and the Color Glass Condensate. 


\subsection{The Energy Density is Big}

The particle multiplicity as a function of energy has been measured at RHIC. Combining the multiplicity data together with the measurements

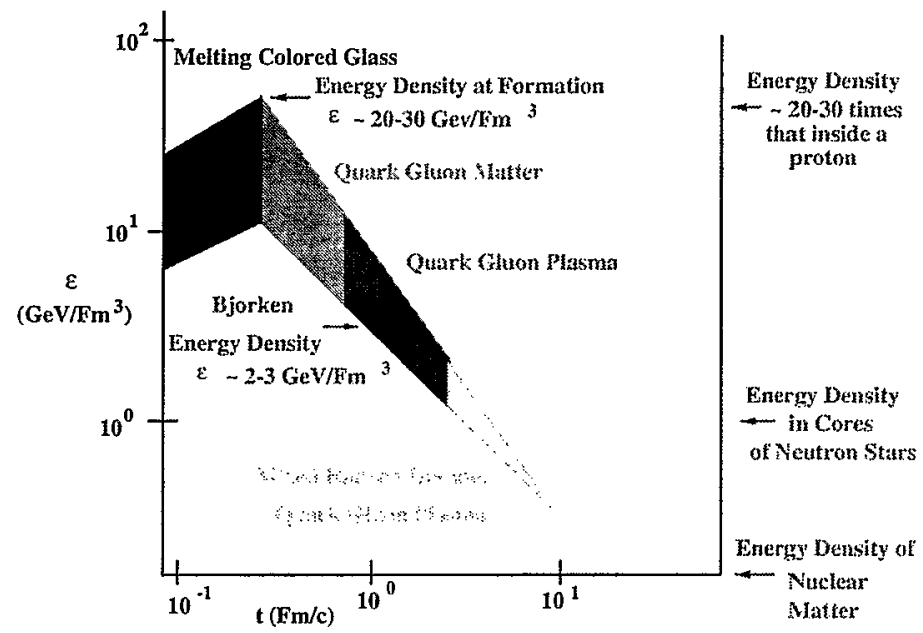

Figure 11. Bounds on the energy density as a function of time in heavy ion collisions.

of transverse energy or of typical particle transverse momenta, one can determine the energy density of the matter when it decouples. One can then extrapolate backwards in time. We extrapolate backwards using 1 dimensional expansion, since decoupling occurs when the matter first begins to expands three dimensionally. We can extrapolate backwards until the matter has melted from a Color Glass.

To do this extrapolation we use that the density of particles falls as $N / V \sim 1 / t$. If the particles expand without interaction, then the energy per particle is constant. If the particles thermalize, then $E / N \sim T$, and since $N / V \sim T^{3}$ for a massless gas, the temperature falls as $T \sim t^{-1 / 3}$. For a gas which is not quite massless, the temperature falls somewhere in the range $T_{0}>T>T_{o}\left(t_{o} / t\right)^{1 / 3}$, that is the temperature is bracketed by the value corresponding to no interaction and to that of a massless relativistic gas. This 1 dimensional expansion continues until the system begins to feel the effects of finite size in the transverse direction, and then rapidly cools through three dimensional expansion. Very close to when three dimensional expansion begins, the system decouples and particles free stream without 
further interaction to detectors.

We shall take a conservative overestimate of this time to be of order $t_{m e l t} \sim .3 \mathrm{Fm} / \mathrm{c}$ The extrapolation backwards is bounded by $\epsilon_{f}\left(t_{f} / t\right)<$ $\epsilon(t)<\epsilon_{f}\left(t_{f} / t\right)^{4 / 3}$. The lower bound is that assuming that the particles do not thermalize and their typical energy is frozen. The upper bound assumes that the system thermalizes as an ideal massless gas. We argued above that the true result is somewhere in between. When the time is as small as the melting time, then the energy density begins to decrease as time is further decreased.

This bound on the energy density is shown in Fig. 11. On the left axis is the energy density and on the bottom axis is time. The system begins as a Color Glass Condensate, then melts to Quark Gluon Matter which eventually thermalizes to a Quark Gluon Plasma. At a time of a few $\mathrm{Fm} / \mathrm{c}$, the plasma becomes a mixture of quarks, gluons and hadrons which expand together.

At a time of about $10 \mathrm{Fm} / \mathrm{c}$, the system falls apart and decouples. At a time of $t \sim 1 \mathrm{Fm} / \mathrm{c}$, the estimate we make is identical to the Bjorken energy density estimate, and this provides a lower bound on the energy density achieved in the collision. (All estimates agree that by a time of order $1 \mathrm{Fm} / \mathrm{c}$, matter has been formed.) The upper bound corresponds to assuming that the system expands as a massless thermal gas from a melting time of $3 \mathrm{Fm} / \mathrm{c}$. (If the time was reduced, the upper bound would be increased yet further.) The bounds on the energy density are therefore

$$
2-3 \mathrm{GeV} / \mathrm{Fm} \mathrm{m}^{3}<\epsilon<20-100 \mathrm{GeV} / \mathrm{Fm}^{3}
$$

where we included a greater range of uncertainty in the upper limit because of the uncertainty associated with the formation time. The energy density of nuclear matter is about $0.15 \mathrm{GeV} / \mathrm{Fm}^{3}$, and even the lowest energy densities in these collisions is in excess of this. At late times, the energy density is about that of the cores of neutron stars, $\epsilon \sim 1 \mathrm{GeV} / \mathrm{Fm}^{3}$.

At such extremely high energy densities, it is silly to try to describe the matter in terms of anything but its quark and gluon degrees of freedom.

In the write up for this talk, there is not space to go through the detailed arguments for the specific things which have been shown at RHIC. In the following enumeration, I will list my opinions about what has been shown, and the interested reader is invited to look at the discussion in for example my talk in Jaipur ${ }^{2}$.

- The Gross Features of Multiplicity Distributions Are Con- 
sistent with Colored Glass Comparison with predictions of the Color Glass Condensate predict the correct dependence of the multiplicity of produced particles on the centrality of the collision. One also correctly predicts the rapidity dependence.

- Matter Has Been Produced which Interacts Strongly with Itself Measurements of the momentum space anisotropies of produced particles as a function of impact parameter can only be explained by having strong, early, and frequent interactions of the quarks and gluons produced in the collision.

- The Single Particle Distributions at Low $p_{T}$ are Consistent with a Thermalized Quark Gluon Plasma. Hydrodynamic computations provide detailed predictions for the spectrum of produced particles. At low $p_{T}<2 \mathrm{GeV}$, these predictions are in quantitative accord with the data.

- The High $p_{T}$ Particle Distributions Hint at New Physics. At high $p_{T}>2 \mathrm{GeV}$, the spectrum of produced particles is depleted relative to what is expected from hard collisions of the constituents of the protons and neutrons in the nuclei. This may be due to rescattering in an exceptionally opaque Quark Gluon Plasma, or it may be due to the effects of the Color Glass Condensate on the initial state nuclear wavefunction. This will be resolved in the $d A$ run at RHIC, since in this environment one can isolate the effects of the initial nuclear wavefunction

- Abundances of Produced Particles are Consistent with that Expected from a Quark Gluon Plasma Thermal models with fixed chemical potential and temperature describe well the abundances of produced species of particles. With a more compelling argument for thermalization, this result may provide a direct measurement of the flavor composition of the Quark Gluon Plasma.

- The Lifetime and Size of the Matter Produced at RHIC Has Been Measured Using like particle correlations, the lifetime and spatial extent at decoupling of the matter produced in heavy ion collisions can be measured. At present, the measurements disagree with theoretical computation. The origin of this discrepancy is not understood, and is the subject of much theoretical work.

In addition to the things we have learned from RHIC, there are a set of issues about which we expect to understand much more in the not too 
distant future. These are:

- Confinement and Chiral Symmetry Restoration Low mass dilepton measurements can determine whether some low mass hadrons have dissociated, due to deconfinement, or have had their mass shifted due to chiral symmetry restoration effects. Low mass dilepton pair measurements are challenging, requiring both good resolution ring imaging Cherenkov detection, and a good direct measurement of charm in order to subtract backgrounds.

- Confinement and $J / \Psi$ Suppression

The measurement of the $J / \Psi$ cross section can teach us about the properties of matter produced in RHIC if one knows how it is produced in $p p$ and $p A$ collisions, and if one has a measure of the direct charm production. If the Quark Gluon Plasma deconfines quarks, there should be no in media production of the $J / \Psi$

\section{Acknowledgements}

I grateful acknowledge conversations with Dima Kharzeev, Robert Pisarski and Raju Venugopalan on the subject of this talk

This manuscript has been authorized under Contract No. DE-AC0298H10886 with the U. S. Department of Energy.

\section{References}

1. For a summary of recent results see the excellent review talk by Jean-Paul Blaizot, Lectures at the 40th Internationale Universitatswochen fuer Theoretische Physik: Dense Matter (IUKT 40), Schladming, Styria, Austria, 3-10 Mar 2001. Published in Lect.Notes Phys.583:117-160,2002 Also in *Schladming 2001, Lectures on quark matter* 117-160; hep-ph/0107131.

2. For a summary of recent results see the excellent review talk by F. Karsch, Lectures at the 40'th Internationale Universitatswochen fuer Theoretische Physik, Dense Matter, Schladming, Styria, Austria, 3-10 March 2001, published in Lect.Notes Phys.583:209-249,2002 Also in *Schladming 2001, Lectures on quark matter* 209-249; hep-lat/0106019.

3. See for example: Edmond Iancu, Andrei Leonidov and Larry McLerran; Lectures given at Cargese Summer School on QCD Perspectives on Hot and Dense Matter, Cargese, France, 6-18 Aug 2001; hep-ph/020227.

4. Larry McLerran; Invited talk at International Conference on Physics and Astrophysics of Quark - Gluon Plasma (ICPAQGP 2001), Jaipur, India, 2630 Nov 2001 ; hep-ph/0202025 
. 